\title{
PREPARATION AND IN VITRO EVALUATION OF METOPROLOL-LOADED BOVINE SERUM ALBUMIN NANOPARTICLES
}

\author{
SHIVA KUMAR YELLANKI*, SAI MANOJ A, MANGILAL T \\ Department of Pharmaceutics, Geethanjali College of Pharmacy, Cheeryal (V), Keesara (M), Hyderabad. Telangana, India. \\ Email: shivakmr19842@gmail.com
}

Received: 15 September 2020, Revised and Accepted: 24 November 2020

\section{ABSTRACT}

Objective: The aim of the present research was to prepare metoprolol-loaded nanospheres. Metoprolol-loaded bovine albumin nanospheres were prepared by nanoprecipitation method. Metoprolol is beta-1-adrenergic receptor inhibitor specific to cardiac cells, thus producing negative chronotropic and ionotropic effect.

Methods: Metoprolol nanospheres were prepared by nanoprecipitation method, using bovine serum albumin as polymer. The prepared nanospheres are evaluated for particle size evaluation, drug entrapment efficiency, and zeta potential. Drug-excipient compatibility was determined using Fouriertransform infrared spectroscopy. In vitro release and solubility of the drug from nanoparticles were determined.

Results: The particle size of prepared metoprolol nanospheres was found to be always less than $200 \mathrm{~nm}$. Maximum particle size was found to be $196 \pm 2.03 \mathrm{~nm}$ of batch 4 nanoparticles. Entrapment efficiency of prepared nanospheres was above $80 \%$ and maximum percentage entrapment efficiency was found to be $80.4 \pm 0.51 \%$. It was found that the percentage entrapment efficiency and drug release were extended with increase in polymer concentration. Zeta potential of the optimized formulation was found to be $-20.4 \mathrm{mV}$. In vitro drug release studies have shown the prolonged release of $94.5 \pm 0.54 \mathrm{up}$ to $10 \mathrm{~h}$. Drug release rate is extended with an increase in polymer concentration.

Conclusion: Results have concluded that the albumin nanospheres loaded with metoprolol have reduced the blood pressure within 24 h and the prepared nanospheres are effective compared to other formulations and drug delivery.

Key words: Metoprolol, Nanospheres, Bovine serum albumin, Nanoprecipitation, Entrapment efficiency, Zeta potential.

(C) 2021 The Authors. Published by Innovare Academic Sciences Pvt Ltd. This is an open access article under the CC BY license (http://creativecommons.org/ licenses/by/4.0/) DOI: http://dx.doi.org/10.22159/ajpcr.2021v14i1.39738. Journal homepage: https://innovareacademics.in/journals/index.php/ajpcr

\section{INTRODUCTION}

Nanospheres are the particles of spherical in shape and size varying between $10 \mathrm{~nm}$ and $200 \mathrm{~nm}$ in diameter. Homogeneous structure in the whole particle is seen in nanospheres. Nanospheres have the drug wrapped in polymer materials. The drug is uniformly dispersed in the nanosphere polymer matrix either by dissolving or entrapment, encapsulation, or attached to the matrix of the polymer [1]. Drug is released from the polymer matrix by any of the three mechanisms:

- Swelling of polymeric matrix because of hydration and resulting in release through diffusion

- Rupture or degradation of polymer at the delivery site by enzymatic reaction and resulting in the release of the drug

- $\quad$ Release of the drug from the polymer by dissociation from the polymer matrix [2].

Nanosphere drug delivery is of more importance in recent years as they exhibit the following benefits such as better encapsulation, improved bioavailability, controlled release and lower toxic effects, and dose proportionality.

Nanospheres or nanoparticles have the ability to deliver drugs in optimum dosage range often resulting in increased therapeutic efficiency of drugs, less side effects, and patient compliance. With these abilities, there has been increased importance for nanospheres and nanoparticles in various fields of medicine [3].

The various fields in which the nanospheres are used are as follows:

- Nanospheres in cancer therapy

- They are used as to treat various lung infections
The purpose of this research work is to formulate metoprolol-loaded bovine serum albumin nanospheres by nanoprecipitation method using bovine albumin as a polymer. Thus, providing improved therapeutic effects with minimal side effects compared to other formulations of metoprolol.

\section{MATERIALS AND METHODS}

Metoprolol was obtained from Zydus Cadila Pharma Ltd. (Gujarat). Polymer bovine serum albumin was obtained from SD Chemicals Ltd. (Hyderabad). Ethanol was obtained from Arene Life Sciences, Hyderabad.

\section{METHODS}

Metoprolol-loaded bovine serum albumin nanospheres were prepared by nanoprecipitation method.

\section{Compatibility studies}

Compatibility studies are performed for any interaction of drug and polymer. This study is performed by Fourier transform infrared (FT-IR). It is an analytical technique used to detect various functional groups of the molecules. FTIR studies help in identification of functional groups present in a mixture of drug and polymer. Interactions of drug product in solid state can be studied by FT-IR spectroscopy.

Samples are mixed with potassium bromide in ratio of 1:100 and placed in sample holder of FTIR and scanned. The spectrum that is obtained of metoprolol sample is compared with the spectrum of metoprolol along with the polymer (albumin) [4]

\section{Differential scanning calorimetry (DSC)}

DSC helps in analyzing the physical state of metoprolol. Temperature range and heat rate are determined by DSC. Before thermal analysis, 
the sample of an optimized formulation is placed in desiccator for $24 \mathrm{~h}$. Five milligrams of the sample were sealed in aluminum crucible and constantly heated at $10^{\circ} \mathrm{C} / \mathrm{min}$ with a temperature range of $25-200^{\circ} \mathrm{C}$. Empty aluminum pan is used as a reference [5].

\section{Calibration curve of Metoprolol}

Metoprolol is analyzed in ultraviolet (UV) spectrophotometer to obtain the standard calibration curve using different concentrations of metoprolol and water as dissolution medium.

\section{Standard stock solution preparation}

In a volumetric flask of $100 \mathrm{ml}$, metoprolol is placed in accurate quantity of $100 \mathrm{mg}$ and the volume is made up to the mark using distilled water. This solution is further made up to a quantity of $1000 \mathrm{ml}$ using distilled water. A $10 \mathrm{ml}$ solution was now taken with the help of a pipette and make up to the volume of $100 \mathrm{ml}$ in a volumetric flask which now indicates the standard solution.

\section{Preparation of metoprolol-loaded bovine serum albumin nanospheres}

Metoprolol-loaded bovine serum albumin nanospheres are prepared by nanoprecipitation method. In this method, the protein (bovine serum albumin) of predetermined quantity is dissolved in required amount of water (aqueous phase). This aqueous polymeric phase is now incorporated with the drug (metoprolol) in a determined quantity and this aqueous polymeric solution containing the drug is subjected to constant stirring at high speed with the help of a propeller. During this high-speed stirring, the organic phase containing an organic solution (ethanol) is injected at the rate of $1 \mathrm{ml} / \mathrm{min}$. Cross-linking agent like glutaraldehyde is used in minute quantity of $1 \mathrm{ml}$.

This constant stirring of aqueous and organic phases at high speed of 700-1000 rpm with propeller, for 90-120 min with glutaraldehyde as a cross-linking agent leads to subsequent precipitation of the organic phase and binding with the polymeric aqueous phase which results in the formation of metoprolol nanospheres. A pictorial representation of nanoprecipitation method is shown in Fig. 1 and Table 1.

Varied number of formulations of metoprolol nanospheres was prepared with different concentrations of polymer (bovine albumin) and different quantities of aqueous phase (water), drug quantity, organic phase quantity, and the cross-linking agent quantity remain the same in all formulations. Varied formulations are prepared to select the optimal formulation and these are tabulated and are shown in Table 1.

Table 1: Different formulations of metoprolol nanospheres

\begin{tabular}{lllllll}
\hline Formulation & F1 & F2 & F3 & F4 & F5 & F6 \\
\hline Metoprolol & $20 \mathrm{mg}$ & $20 \mathrm{mg}$ & $20 \mathrm{mg}$ & $20 \mathrm{mg}$ & $20 \mathrm{mg}$ & $20 \mathrm{mg}$ \\
Ethanol & $50 \mathrm{ml}$ & $50 \mathrm{ml}$ & $50 \mathrm{ml}$ & $50 \mathrm{ml}$ & $50 \mathrm{ml}$ & $50 \mathrm{ml}$ \\
Water & $50 \mathrm{ml}$ & $75 \mathrm{ml}$ & $100 \mathrm{ml}$ & $50 \mathrm{ml}$ & $75 \mathrm{ml}$ & $100 \mathrm{ml}$ \\
Albumin & $2 \%$ & $2 \%$ & $2 \%$ & $3 \%$ & $3 \%$ & $3 \%$ \\
Glutaraldehyde & $1 \mathrm{ml}$ & $1 \mathrm{ml}$ & $1 \mathrm{ml}$ & $1 \mathrm{ml}$ & $1 \mathrm{ml}$ & $1 \mathrm{ml}$ \\
\hline
\end{tabular}

Table 2: Percentage entrapment efficiency of formulations $(\mathrm{SD} \pm \mathrm{n}=3)$

\begin{tabular}{lll}
\hline S. No. & Formulation code & \%EE $\mathbf{S} . \mathbf{D}$ \\
\hline 1 & F1 & $68.7 \pm 0.56$ \\
2 & F2 & $74.1 \pm 0.24$ \\
3 & F3 & $71.2 \pm 0.35$ \\
4 & F4 & $80.4 \pm 0.51$ \\
5 & F5 & $74.1 \pm 0.25$ \\
6 & F6 & $75.3 \pm 0.32$ \\
\hline
\end{tabular}

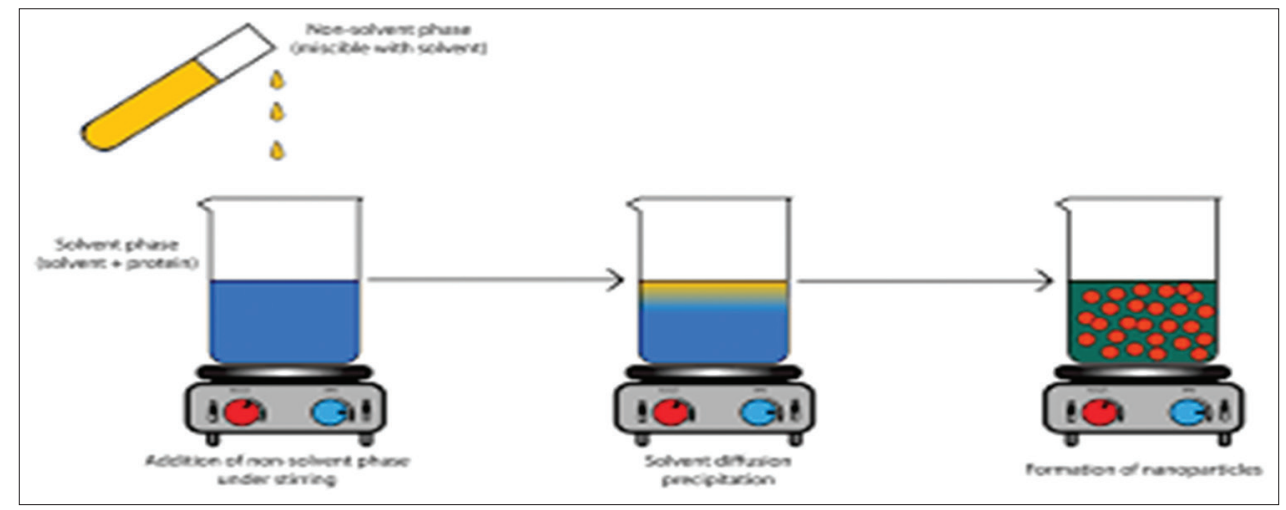

Fig. 1: Representation of nanoprecipitation method

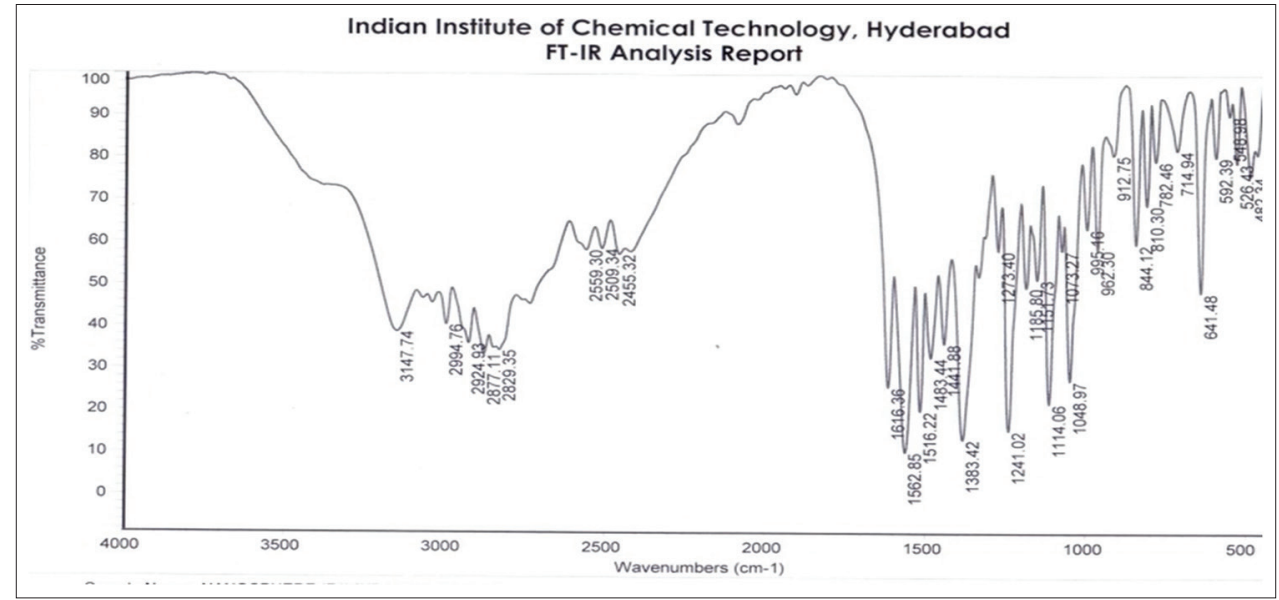

Fig. 2: Fourier transform infrared image of metoprolol 
Table 3: In vitro $\%$ drug release of formulations ( $\mathrm{SD} \pm \mathrm{n}=3$ )

\begin{tabular}{|c|c|c|c|c|c|c|}
\hline Time (h) & F1 & F2 & F3 & F4 & F5 & F6 \\
\hline 0 & 0 & 0 & 0 & 0 & 0 & 0 \\
\hline 1 & $14.1 \pm 0.24$ & $14.5 \pm 0.22$ & $15.1 \pm 0.1$ & $16.5 \pm 0.23$ & $15.9 \pm 0.52$ & $14.8 \pm 0.21$ \\
\hline 2 & $21.4 \pm 0.31$ & $22.1 \pm 0.31$ & $21.8 \pm 0.24$ & $22.4 \pm 0.57$ & $21.9 \pm 0.63$ & $21.6 \pm 0.15$ \\
\hline 3 & $28.5 \pm 0.24$ & $29.1 \pm 0.11$ & $28.4 \pm 0.15$ & $29.7 \pm 0.63$ & $28.4 \pm 0.14$ & $27.4 \pm 0.24$ \\
\hline 4 & $32.4 \pm 0.57$ & $30.2 \pm 0.53$ & $31.4 \pm 0.55$ & $36.5 \pm 0.54$ & $31.4 \pm 0.25$ & $30.6 \pm 0.57$ \\
\hline 5 & $46.1 \pm 0.31$ & $45.1 \pm 0.67$ & $44.2 \pm 0.84$ & $45.9 \pm 0.15$ & $43.1 \pm 0.15$ & $44.2 \pm 0.64$ \\
\hline 6 & $58.4 \pm 0.14$ & $60.5 \pm 0.74$ & $59.7 \pm 0.45$ & $60.9 \pm 0.26$ & $60.6 \pm 0.24$ & $58.9 \pm 0.62$ \\
\hline 8 & $75.4 \pm 0.51$ & $78.4 \pm 0.64$ & $79.5 \pm 0.85$ & $80.2 \pm 0.54$ & $78.9 \pm 0.12$ & $79.2 \pm 0.31$ \\
\hline 9 & $81.3 \pm 0.72$ & $85.1 \pm 0.14$ & $86.7 \pm 0.16$ & $87.4 \pm 0.45$ & $86.2 \pm 0.25$ & $86.7 \pm 0.24$ \\
\hline 10 & $87.1 \pm 0.36$ & $91.2 \pm 0.27$ & $92.3 \pm 0.25$ & $94.5 \pm 0.54$ & $90.1 \pm 0.61$ & $94.1 \pm 0.71$ \\
\hline
\end{tabular}

\section{RESULTS}

\section{Scanning electron microscopy (SEM)}

SEM helps in visualizing the morphology of prepared nanospheres where the water suspended nanospheres are mounted on the holder of the SEM. These are subjected to gold sputtering and the using the double side carbon tape mounted on aluminum stud, the slide was attached to the microscope. By operating at voltage of $20 \mathrm{kV}$ electron beam at desired magnification, the photomicrographs of nanospheres were captured [6].

The SEM photomicrographs of sample F4 are shown in Fig. 2. These photographs revealed that the nanospheres are smooth and round uniform in nature (Fig. 3)

\section{FT-IR}

When the samples of metoprolol and the optimized formulation of metoprolol and polymer are subjected to FTIR, peaks were observed in IR spectroscopy for both the samples.

FTIR spectra of metoprolol (pure drug) have shown the peaks at $3147.74 \mathrm{~cm}^{-1}, 2994.76 \mathrm{~cm}^{-1}, 2924.93 \mathrm{~cm}^{-1}$, and $2877.11 \mathrm{~cm}^{-1}$. Spectra of the optimized formulation which consists of the drug and polymer (metoprolol and albumin) have shown the peaks at $3149.71 \mathrm{~cm}^{-1}$, $2994.76 \mathrm{~cm}^{-1}, 2925.91 \mathrm{~cm}^{-1}$, and $2877.26 \mathrm{~cm}^{-1}$. This indicates that there are no major interactions between pure drug (metoprolol) and the formulation (albumin and metoprolol) concluding that the drug and excipients are compatible with each other.

DSC

The optimized formulation of metoprolol (pure drug) and the optimized formulation (drug and polymer) are subjected to DSC.

The pure metoprolol drug has shown a sharp peak at $126.29^{\circ} \mathrm{C}$ and almost similar endothermic changes were observed by optimized formulation as it shows a peak at a value of $140.31^{\circ} \mathrm{C}$ indicating that there are no major interactions between the drug and excipients which concludes that the pure drug (metoprolol) and the polymer are compatible.

\section{Entrapment efficiency}

The amount of drug entrapped into the prepared nanospheres was determined by entrapment efficiency. In this process, certain weighed amount of nanospheres $(10 \mathrm{mg})$ is added to $10 \mathrm{ml}$ of distilled water and then centrifugation was performed and $10 \mathrm{ml}$ supernatant liquid was collected and added with $4 \mathrm{ml}$ of methanolic solution. This is finally analyzed by UV spectrophotometer and results are obtained [7].

The formulation of code F4 is found to show an entrapment efficiency of $80.4 \pm 0.51 \%$ (Table 2 ).

\section{In vitro drug release studies}

Franz diffusion cell is utilized for the in vitro drug release studies of prepared metoprolol formulations. Phosphate buffer is used as dissolution medium and is carried out for $10 \mathrm{~h}$ (Table 3). The drug release is by diffusion or degradation. It is found that the drug release from all the formulations is higher than the pure drug and the

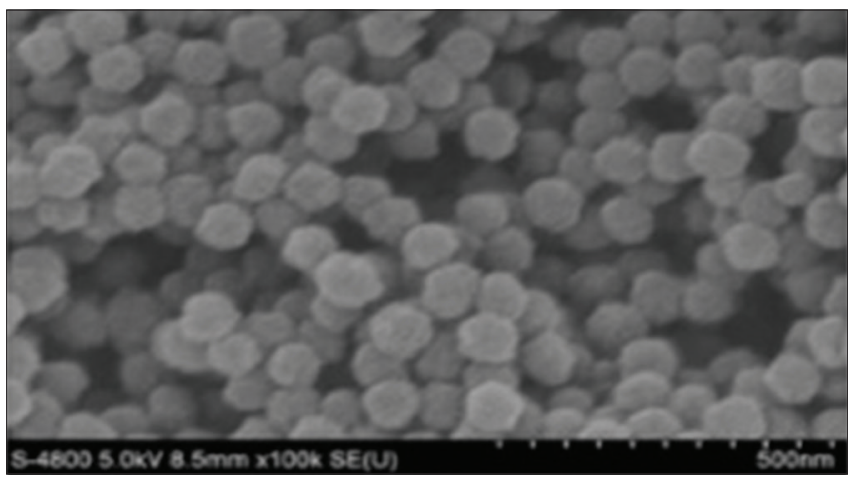

Fig. 3: Scanning electron microscopy photomicrographs of nanosphere formulation F4

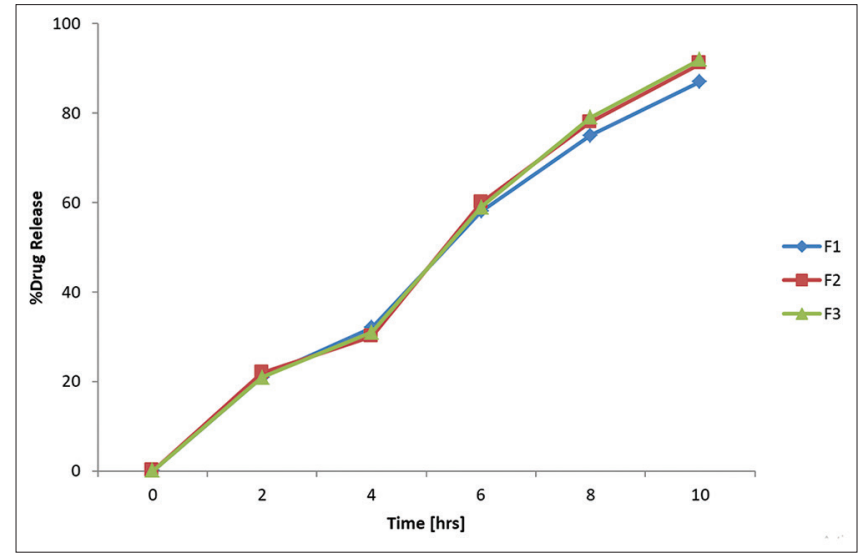

Fig. 4: Graphical representation of $\%$ drug release of formulations $F 1, F 2$, and F3

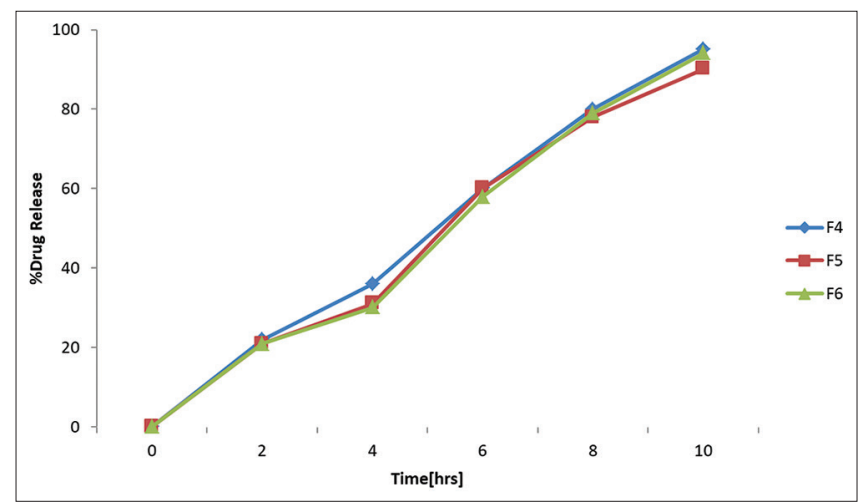

Fig. 5: Graphical representation of $\%$ drug release of formulations F4, F5, and F6 
Table 4: Zeta potential values of prepared formulations

\begin{tabular}{lll}
\hline S. No. & Formulation code & Zeta potential $(\mathbf{m V})$ \\
\hline 1 & F1 & -17.1 \\
2 & F2 & -19.6 \\
3 & F3 & -17.9 \\
4 & F4 & -20.4 \\
5 & F5 & -20.2 \\
6 & F6 & -21.7 \\
\hline
\end{tabular}

formulation with code $\mathrm{F} 4$ has been noticed to show a maximum drug release of $94.5 \pm 0.54$ in $10 \mathrm{~h}$ and followed first-order kinetics along with Higuchi model. Drug release from all formulations is depicted in Table 4 and Figs. 4,5.

\section{Zeta potential}

The dominant component of particle surface can be known by zeta potential. Zeta potential characterizes the charge on surface particles, thus playing an important role in determining the stability.

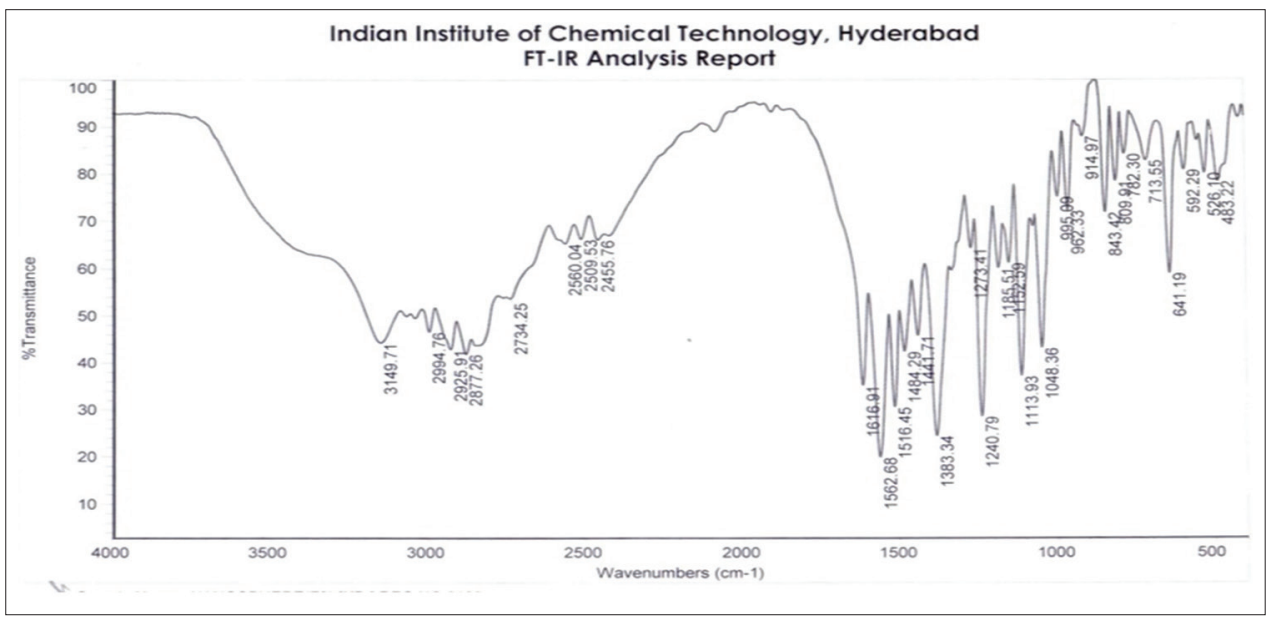

Fig. 6: Fourier transform infrared image of optimized formulation (metoprolol and albumin)

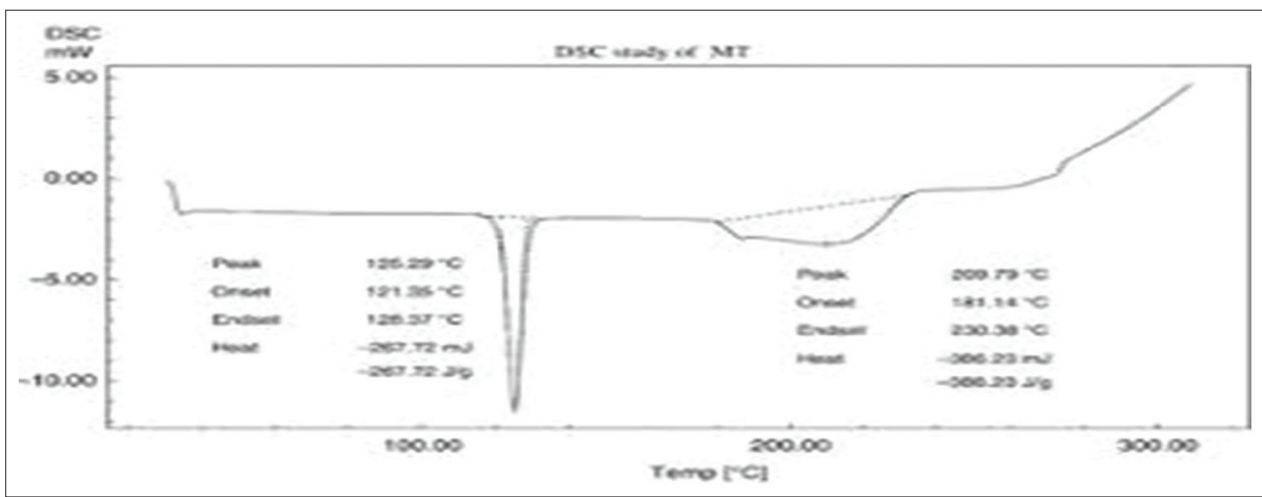

Fig. 7: Differential scanning calorimetry graph of metoprolol

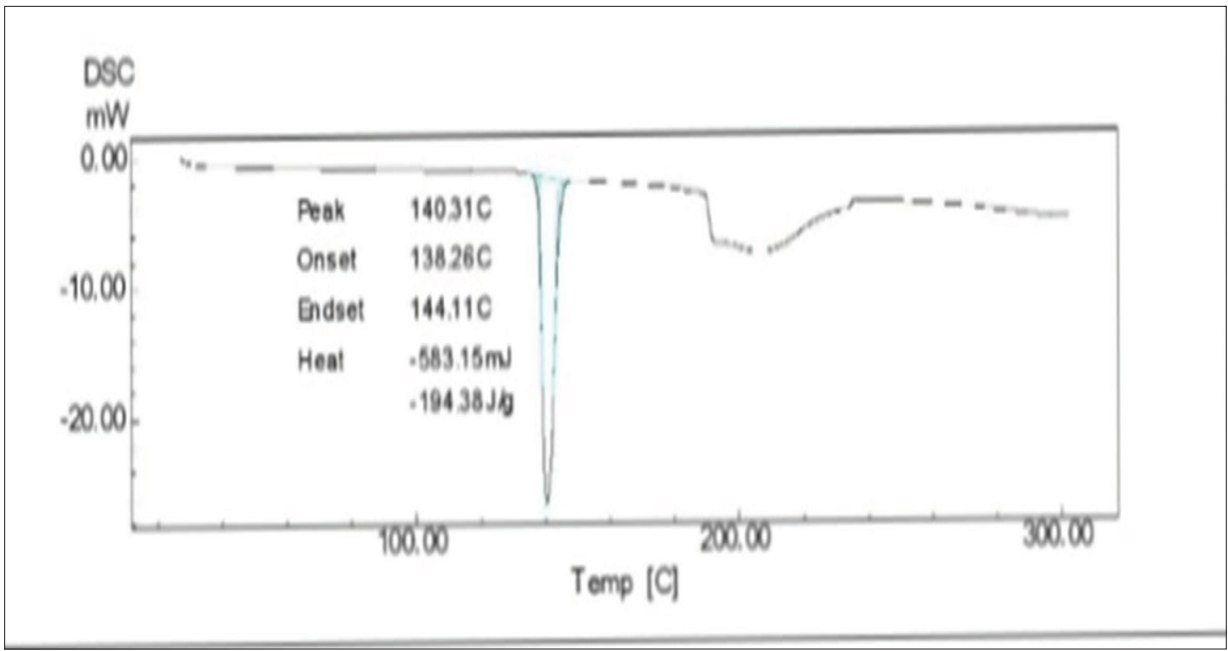

Fig. 8: Differential scanning calorimetry graph of optimized formulation 


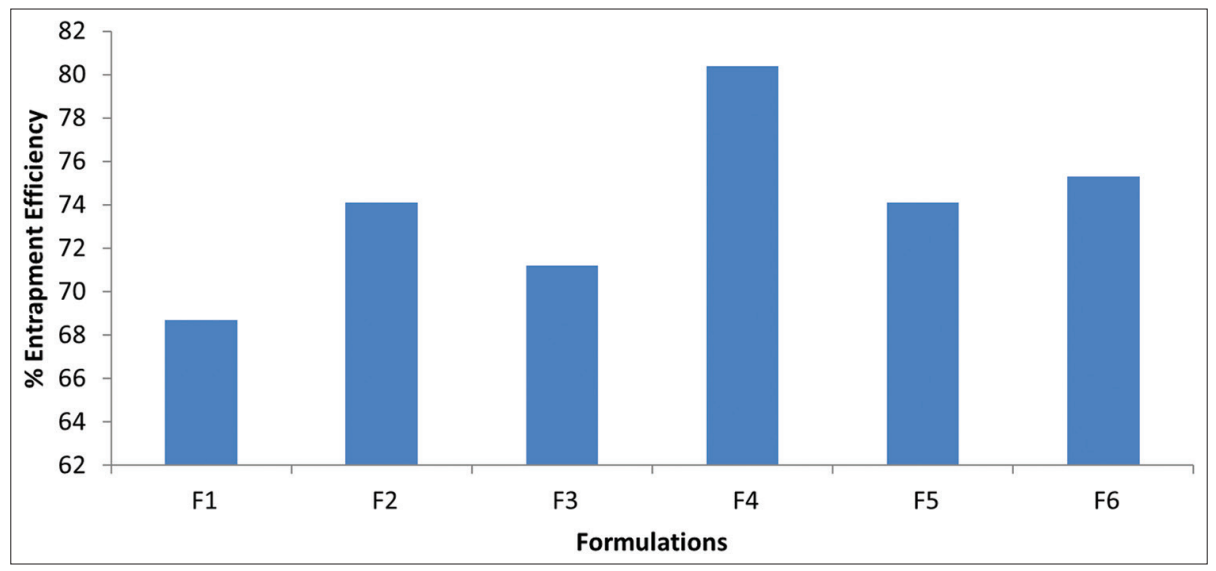

Fig. 9: Graphical representation of \% entrapment efficiency of formulations

Zeta potential is predominantly measured using Zetasizer Nano ZS 90 (Malvern Instruments, UK) by determination of electrophoretic mobility by performing electrophoresis experiment on the sample. Now, Henry equation was used to determine the zeta potential of formulations F1-F6 [8]. Zeta potential of prepared metoprolol nanospheres is important to determine the stability of nanospheres and the obtained zeta potential values for prepared formulations are mentioned in Table 4. The zeta potential of optimized formulation F4 was found to be $-20.4 \mathrm{mV}$.

\section{DISCUSSION}

FT-IR and DSC studies were performed to observe any interaction between the drug and polymer which can be reflected by the change in position of peak of metoprolol from Figs 6 and 7 of FT-IR and from Figs. 8 and 9 of DSC, it is clear that there are no interactions between metoprolol and polymer bovine serum albumin. The amount of drug entrapped in the nanospheres is measured by entrapment efficiency and the formulation $\mathrm{F} 4$ has shown the maximum entrapment efficiency of $80.4 \pm 0.51 \%$. Zeta potential of the optimized formulation F4 was found to be $-20.4 \mathrm{mV}$.

It is found from in vitro studies that the drug releases from all the formulations are higher than the pure drug and the formulation with code $\mathrm{F} 4$ have been noticed to show a maximum drug release of $94.5 \pm 0.54$ in $10 \mathrm{~h}$ and followed first-order kinetics along with Higuchi model.

The drug release from the prepared formulations can be ranked in descending order as $\mathrm{F} 4>\mathrm{F} 6>\mathrm{F} 5>\mathrm{F} 3>\mathrm{F} 2>\mathrm{F} 1$.

\section{CONCLUSION}

The present research work deals with the preparation and optimization of metoprolol nanospheres. These nanosphere formulations are optimized based on the drug release and the size characteristics. Among the prepared formulations, the formulation batch nanospheres of F4 are found to be round in size and smooth in nature with the size below $200 \mathrm{~nm}$. The F4 formulation is also found to show high in vitro drug release compared to other formulations and other modes of administration of metoprolol. Zeta potential of optimized formulation was found to be $-20.4 \mathrm{mV}$. Thus, successful attempt was made in preparation of metoprolol-loaded bovine serum albumin nanospheres.

\section{AUTHORS' CONTRIBUTIONS}

The idea of this research work was set by the guidance of Associate Professor, Dr. Y. Shiva Kumar. The research work analyzed by A. Sai Manoj.

\section{AUTHORS' FUNDING}

Nil.

\section{CONFLICTS OF INTEREST}

None.

\section{REFERENCES}

1. Garg G, Sharma PK, Singh A. Nanospheres: A novel approach for targeted drug delivery system a review. Int J Pharm Sci Rev Res 2010;5:15.

2. Karuppusamy C, Venkatesan P. Role of nanoparticles in drug delivery system: A comprehensive review. J Pharm Sci Res 2017;9:318-25.

3. Khan I, Saeed L, Khan I. Nanoparticles: Properties, applications and toxicities: A review. Arab J Chem 2017;10:1-11.

4. Srinivas P, Preeti KS. Formulation and evaluation of gemcitabine hydrochloride loaded solid lipid nanoparticles. J Glob Trends Pharm Sci 2017;5:2017-23

5. Kumar DP, Subas CD, Subrata C, Soumen R. Formulation and evaluation of solid lipid nanoparticles of a poorly water soluble model drug, ibuprofen-A research article. Int J Pharm 2012;3:2230-8407.

6. Ekambaram P, Abdul HS. Formulation and evaluation of solid lipid nanoparticles of ramipril. J Young Pharm 2011;3:216-20.

7. Dora CP, Singh SK, Kumar S, Datusalia AK, Deep A. Development and characterization of nanoparticles of glibenclamide by solvent displacement method. Acta Pol Pharm 2010;67:283-90.

8. Dinda A, Biswal I, Chowdhury P, Mohapatra R. Formulation development and evaluation of paclitaxel loaded solid lipid nanoparticles using glyceryl monostearate. J Appl Pharm Sci 2013;3:133-8. 